\title{
Gesellschaftsnachrichten
}

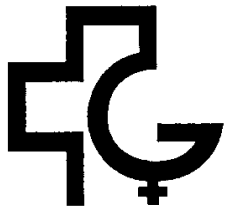

\section{Schweizerische Gesellschaft für Gynäkologie und Geburtshilfe}

http://www.sggg.ch

\section{Präsident}

Dr. med. Lucio Bronz

Ospedale Regionale Bellinzona e Valli

CH-6500 Bellinzona

Tel. +41918119145, Fax +41918119042

E-Mail lucio.bronz@eoc.ch

\author{
Sekretär \\ Prof. Dr. med. Mario Litschgi \\ Kantonsspital Schaffhausen \\ CH-8208 Schaffhausen (Schweiz) \\ Tel. +41526342 315, Fax +41526342398 \\ E-Mail Mario.litschgi@kssh.ch
}

\section{Liebe Kolleginnen und Kollegen}

Der Vorstand der SGGG hat an seiner Sitzung vom 16. Januar 2004 die Unsicherheiten bei den Mitgliedern nach der Tarmed-Einführung wahrgenommen.

Wir möchten zuerst festhalten, dass die 3 Schwerpunkttitel (gynäkologische Onkologie, Geburtshilfe und fetomaternale Medizin, Reproduktionsmedizin und gynäkologische Endokrinologie) nichts mit Tarmed zu tun haben! Das heisst, dass ein FMH-Gynäkologe und -Geburtshelfer alle im Tarmed aufgeführten, inklusive die überhöhten Leistungen während der nächsten 3 Jahre verrechnen kann. Die neue Fortbildungsordnung verlangt, dass innerhalb von den nächsten 3 Jahren jeder Arzt 150 Credits an Fortbildung nachweist, damit seine Leistungen weiterhin von den Krankenkassen bezahlt werden.

Es ist die Aufgabe unserer Akademie, Ihnen die in diesen 3 Jahren nötigen Fortbildungen aufzuzeigen, deren Besuch obligatorisch ist, damit Sie weiterhin voll arbeiten können. Ihren Wunsch nach einem vereinfachten Beurteilungsbogen der Fortbildungen wird die Akademie bald erfüllen.

Für die Rezertifizierung des Fähigkeitsausweises «Schwangerschafts-Ultraschall» werden voraussichtlich nur 15 Credits innerhalb von 5 Jahren verlangt, was leicht zu erreichen sein sollte. Wir glauben damit dem Wunsch, welcher an der Generalversammlung in Lugano 2003 laut wurde, entsprochen zu haben.

Wir werden Laser-Kurse organisieren, damit es auch ohne den Fähigkeitsausweis «Laser» der FMS möglich sein wird, weiterhin Laser im gynäkologischen Bereich anwenden und verrechnen zu können.

Der Fähigkeitsausweis «dosisintensives Röntgen» ist im Besitz von allen Gynäkologen FMH und muss nicht extra verlangt werden. Auch für seine Rezertifizierung werden genaue Erklärungen bekannt gegeben.

Dem Vorstand der SGGG ist bewusst, dass viele Mitglieder beunruhigt und empört sind, wie sie jetzt nach vielen Jahren Praxiserfahrung von der FMH wie Kleinkinder behandelt werden. Wir werden alles daran setzen, dass Sie Ihren Beruf unter den neuen Bedingungen weiter ausüben können. Wir werden Sie darüber entsprechend informieren.

Dr. Lucio Bronz

Präsident der SGGG

Prof. Dr. Mario Litschgi

Generalsekretär der SGGG

\section{KARGER}

Fax +4161306 1234 